\title{
LA ESTELA 1 DE LA MOJARRA
}

Fernando Winfield Capitaine

A mediados del mes de noviembre de 1986 fue rescatada del lecho del río Acula, después de siglos de inmersión, una portentosa estela que contiene 437 glifos agrupados en 21 columnas o cartuchos verticales con un personaje riquísimamente ataviado.

Procede de La Mojarra, municipio de Alvarado, zona ubicada entre los sitios arqueológicos de Cerro de las Mesas (municipio de Tlalixcoyan) y Tres Zapotes (municipio de Santiago Tuxtla), en el estado de Veracruz, México.

La estela presenta secciones destruidas por la mano del hombre, quizá por miembros de la sociedad enemiga de la cultura que hizo posible el trabajo lapidario y erección de la piedra, mismos que la arrojaron al fondo del río. Tomando en consideración el tamaño de los glifos y los espacios rotos, se hizo la consideración de que 28 glifos desaparecieron en el conjunto " $B$ ", lo cual suma un total de 465 glifos inscritos originalmente, por lo que dicha estela se convierte en la que contiene el mayor número de glifos descubierta en el continente americano.

Aparte de esta relevancia numérica, adquiere una característica sumamente excepcional, consistente en que nadie ha explicado el significado de la mayoría de los glifos que presenta en la casi totalidad de su superficie.

Miguel Covarrubias (p. 118) publicó los dibujos que aparecen incisos en la Estatuilla de Tuxtla, anotando que están sin descifrar. Más adelante indica que "es uno del los tesoros del Museo Nacional de Estados Unidos en Washington, representa a un personaje, gordo, calvo, jovial que lleva puestos un par de alas y una rara máscara en la boca que se parece al pico de un pato. En sus cuatro costados presenta columnas de glifos, que todavía no han sido descifrados, excepto la fecha, 162 d.C., escrita también con barras y puntos". (p. 120). Dicho objeto mide $20 \mathrm{~cm}$. de alto; está elaborado en jadeíta y representa a un personaje zooantropomorfo, obeso, con una especie de máscara de pato, probable representación de Quetzalcóatl en su advocación de Ehécatl o Dios del Viento. A lo largo de sus cuatro lados la Estatuilla de Tuxtla presenta una serie de glifos en finas incisiones, 20 de las cuales se encuentran en la estela de La Mojarra.

Dichos glifos, numerados en orden descendente, son los siguientes: 
FRENTE: 1,3 ['casa"] , 4, 5 (lámina 1).*

ADVERSO: 2, 6, 7, 8 (lámina 2).

LADO IzQuIERDo: 1 [glifo 715], 3 ["sacrificio"], 7, 9, 12 [glifo solar] (lámina 3) , 14 ["altar"], 15, 16 [glifos "flor" y "sol"], 17, 19 (lámina 4), 24 (lámina 5).

LADO DERECHO: 2 [glifo "C" zapoteca] (lámina 5).

Este hecho vincula el parentesco cultural de la Estatuilla de Tuxtla con la Estela 1 de La Mojarra que forma parte de la colección del Museo de Antropología de la Universidad Veracruzana, sito en la ciudad de Xalapa, capital del estado de Veracruz.

La estela es de basalto y pesa 4 toneladas. Mirándola hacia el frente tiene en la parte izquierda una altura de $234 \mathrm{~cm}$; altura derecha de 210 $\mathrm{cm}$; base de $110 \mathrm{~cm}$, y en la parte superior $142 \mathrm{~cm}$.

El ángulo superior izquierdo tiene un espesor inicial de $54 \mathrm{~cm}$; el derecho, de $15 \mathrm{~cm}$.

El ángulo inferior izquierdo inicia con un espesor de $17 \mathrm{~cm}, y$ termina en el derecho con $45 \mathrm{~cm}$.

El espesor del lado derecho, de arriba hacia abajo, presenta un parámetro que va desde los 15 hasta los $17 \mathrm{~cm}$.

Es decir, el mayor espesor se da en la parte izquierda (de arriba hacia abajo) iniciando con $54 \mathrm{~cm}$, y terminando con $45 \mathrm{~cm}$. Va adelgazándose hacia la derecha de los 15 a los $17 \mathrm{~cm}$.

La lectura de los numerales mayas según la cuenta larga arroja dos fechas: 142 y $153 \mathrm{~d}$. C., lo cual le confiere mayor antigüedad que la que tiene la de Tuxtla.

¿Qué civilización elaboró esta portentosa estela? es una pregunta difícil de responder por el momento. Las fuentes históricas recopiladas por los frailes del siglo XVI no alcanzan a llegar hasta el siglo II $\mathrm{d}$. C.

Por las semejanzas estilísticas que guarda con las estelas del centro de Veracruz (especialmente las de Cerro de las Mesas y de Alvarado), pertenece al grupo. Se ven influencias mayas en el personaje ricamente elaborado y en los numerales, pero también es conveniente advertir la presencia de elementos culturales procedentes de Oaxaca, especialmente de la cultura zapoteca y teotihuacanos. El área en que se localizó la estela es vecina de una que aún en la actualidad se conoce con el nombre de La Mixtequilla.

* Agradezco a Fernando N. Winfield Reyes la elaboración de los dibujos que acompañan este trabajo, obtenidos de una calca realizada por Amador Arguielles; además, la discusión relativa. A Héctor Cuevas Fernández la toma de diapositivas que permitieron corregir los trazos de los glifos. 
En Cerro de las Mesas los personajes inscritos en las estelas representan a sacerdotes o sus deidades, con tocados completamente elaborados cuyas características no se dan más que en la zona maya, pero con diferencias respecto a ésta en el tipo físico y en los elementos que configuran el vestido. Siempre miran hacia su derecha, en tanto que el personaje de la Estela 1 ve hacia su izquierda.

Los glifos de la estela tienen su sello propio. Algunas excepciones son semejantes o idénticas a elementos de escrituras maya y zapoteca; otros aparecen en documentos coloniales del siglo XVI, tal como el que significa la milpa o campo de maíz en el Códice de Santa María Asunción (Williams, p. 31), revelando una asombrosa sobrevivencia de 14 siglos en el pensamiento indígena.

No obstante, estamos ante un nuevo (en el sentido de no conocido anteriormente) sistema de escritura que dejó de utilizarse.

Otros elementos gráficos que aparecen en códices mayas (Vollemaere, 7:267-280) son los marcados con el número 85, 121 (p. 274), 160 (p. 275), pronombre personal de tercera persona de singular, pronombre personal de tercera persona de plural (p. 279).

La revisión del Catálogo de jeroglíficos mayas publicado por Thompson (1962) llevó a la localización e identificación de otros signos de escritura y que aparecen en la estela de La Mojarra.

136 (afijo) (p. 53).

190 (afijo) (p. 59).

216 (afijo) (p. 60).

248 (p. 63), encontrado en un vaso de carey procedente de Uaxactún.

503 (p. 98), Ik, mismo que asigna Vollemaere con el número 85 citado anteriormente.

510 (p. 108), Lamat Venus, introductorio al mes Yax.

511 (p. 110), Muluc, dia en Yaxchilan.

512 (p. 111), Muluc-Burden.

527 (p. 133), Etz’nab, el más estéril de todos los signos de los días.

537 (p. 151) , Xipe.

546 (p. 160), variante de Kin.

561 (p. 174), cielo.

573 (p. 198), Hel, que significa cambio o rotación.

593 (p. 220), jarras o calabazas de los dioses de la lluvia. Tiene el glifo, además, la representación de uno de los dos elementos que integran el concepto de turquesa.

595 (p. 221), algodón. Puede referirse, asimismo, al concepto "desollado". 
607 (p. 230), símbolo del jade.

638 (p. 250), Lamat yucateco.

715 (p.'307) [ijade?].

La estela tiene una combinación de signos abstractos y naturales. Los primeros hacen sumamente difícil su interpretación; probablemente se trata de ideogramas en donde un elemento basta para ofrecer un concepto lingüístico más amplio de lo que representa.

Los esgrafiados con representaciones naturales indican varias clases de seres y objetos. Por ejemplo:

\begin{tabular}{lccc}
\hline \multicolumn{1}{c}{ MOTIVo } & COLUMNA & GLIFO & LÁMINA \\
\hline Personaje masculino & 7 & 17 & 6 \\
Animal degollado & 8 & 15 & 6 \\
Vasijas ceremoniales & 3 & 39 & 6 \\
Serpiente & 9 & 9 & 6 \\
Dios Chaac & 7 & 5 & 7 \\
Dignatario & 5 & $15 \mathrm{a} 17$ & 8 \\
Jaguar & 4 & 16 & 9 \\
Mandíbula (Conjunto & & & \\
$\quad$ "A') & 11 & 7 & 9 \\
Personaje masculino & & 21 & 10 \\
$\quad$ barbado o anciano & 2 & 12 & 10 \\
& 4 & 42 & 10 \\
Cabeza de Tortuga & 4 & 31 & 11 \\
Conejo & 3 & 51 & 11 \\
\hline
\end{tabular}

Los glifos se encuentran dispuestos en dos conjuntos. Mirando de frente a la estela, hay un primero de 12 columnas cuyos elementos se encuentran orientados de izquierda a derecha: se llamará "A".

El segundo conjunto está formado de 9 columnas en las cuales los glifos están desarrollados de derecha a izquierda; se denominará " $B$ ".

Tal disposición afecta el orden de lectura de los símbolos. Es de lógica elemental que los glifos tienen una secuencia de arriba hacia abajo. Aunque algunos autores sostienen qư el orden de lectura en estelas debe ser de arriba hacia abajo y luego de derecha a izquierda (pues tal era el procedimiento seguido por los indígenas en el periodo Post-clásico), la circunstancia de que los dos conjuntos de nuestra estela se oponen (es decir, se en- 
frentan o "miran"), hace que consideremos que la lectura del conjunto de la izquierda o " $A$ ", debe de leerse de arriba hacia abajo y luego de izquierda a derecha para obtener la secuencia correcta, en tanto que el conjunto de la derecha o " $\mathrm{B}$ " deberá de leerse desde arriba hacia abajo y de derecha a izquierda.

Tal procedimiento tendrá efecto en la numeración progresiva de las com lumnas o cartuchos, más no en la de los glifos, pues en este caso siempre es de arriba hacia abajo.

Para mayor claridad en la exposición, el conjunto " $A$ " está dispuesto arriba del personaje principal, representado en escala natural, mientras que el " $B$ " se halla situado frente al personaje, con un desarrollo vertical desde el extremo superior al inferior en la estela.

Abajo se refiere el inventario de columnas con el número correspondiente de glifos en cada conjunto. El "B" tiene 28 glifos destruidos.

\begin{tabular}{|c|c|c|c|}
\hline \multicolumn{4}{|c|}{ COLUMNA } \\
\hline & $\begin{array}{c}\text { CONI UNTO } \\
\text { "A" }\end{array}$ & $\begin{array}{l}\text { CONI UNTO } \\
\text { "B" } \mathrm{B} \text { " }\end{array}$ & DESTRUIDOS \\
\hline 1 & 8 & 14 & \\
\hline 2 & 7 & 46 & \\
\hline 3 & 7 & 53 & \\
\hline 4 & 6 & 51 & \\
\hline 5 & 4 & 49 & \\
\hline 6 & 7 & 51 & 5 \\
\hline 7 & 6 & 50 & 8 \\
\hline 8 & 4 & 51 & 15 \\
\hline 9 & 6 & \multicolumn{2}{|c|}{17 (Fecha maya: $8.5 .16 .9 .7 .[153 \mathrm{~d}$. C.] ). } \\
\hline 10 & 8 & & \\
\hline 11 & 9 & \\
\hline 12 & 11 (Fecha maya: 8.5.3.3.13. [142 d. C.]) & & \\
\hline TOTAL & 83 & $+382=465-$ & $28=437$ \\
\hline
\end{tabular}

Un aspecto que arroja la lectura tiene relación con la circunstancia de que el contenido de una columna de información que finaliza al extremo inferior, funciona como una especie de punto y aparte. Asimismo, a pesar de terminar un mensaje en cada columna, hay una sucesión de hechos relacionados en las columnas vecinas. También es posible que el conjunto " $\mathrm{A}$ " 
integre un momento histórico del pueblo que realizó las inscripciones en la estela, de la misma manera que el "B" y cuya suma forma el todo histórico.

$\mathrm{El}$ orden de lectura propuesto tiene una poderosa lógica interna: cada conjunto finaliza con una fecha, a manera de conclusión.

\section{OBRAS CONSULTADAS}

Caso, Alfonso, "Calendario y escritura de las antiguas culturas de Monte Albán". pp. 115.143, en tomo I de las Obras Completas de Miguel Othón de Mendizábal, México, 1946.

Caso, Alfonso, Los calendarios prehispánicas, México, Universidad Nacional Autónoma de México, Instituto de Investigaciones Históricas, 1967. Covarrubias, Miguel, El Sur de México, México, Instituto Nacional Indigenista, 1980.

Thompson, J. Eric S., A Catalog of Maya Hieroglyphs, Norman University of Oklahoma, 1962.

Vollemaere, Antoon, "Synthèse de paléographie des codex mayas", en Actes du X.LIIe Congrès International des Américanistes, 1979, vol. 7:267-280.

Williams, Barbara J., "Nahuatl soil glyphs from the Codice de Santa María Asunción", en Actes du XLIIe Congrès International des Américanistes, vol. 7:27-37. 


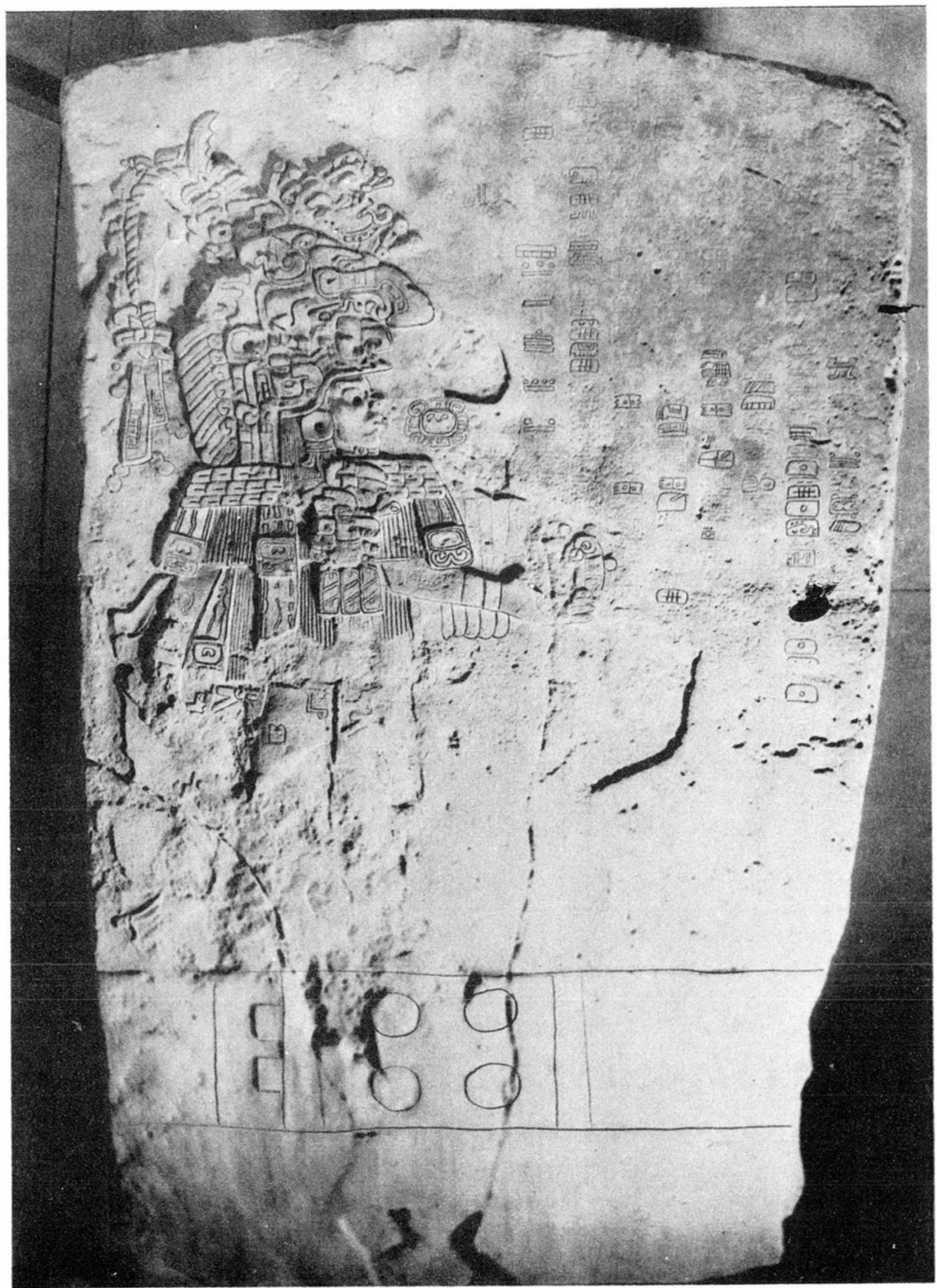



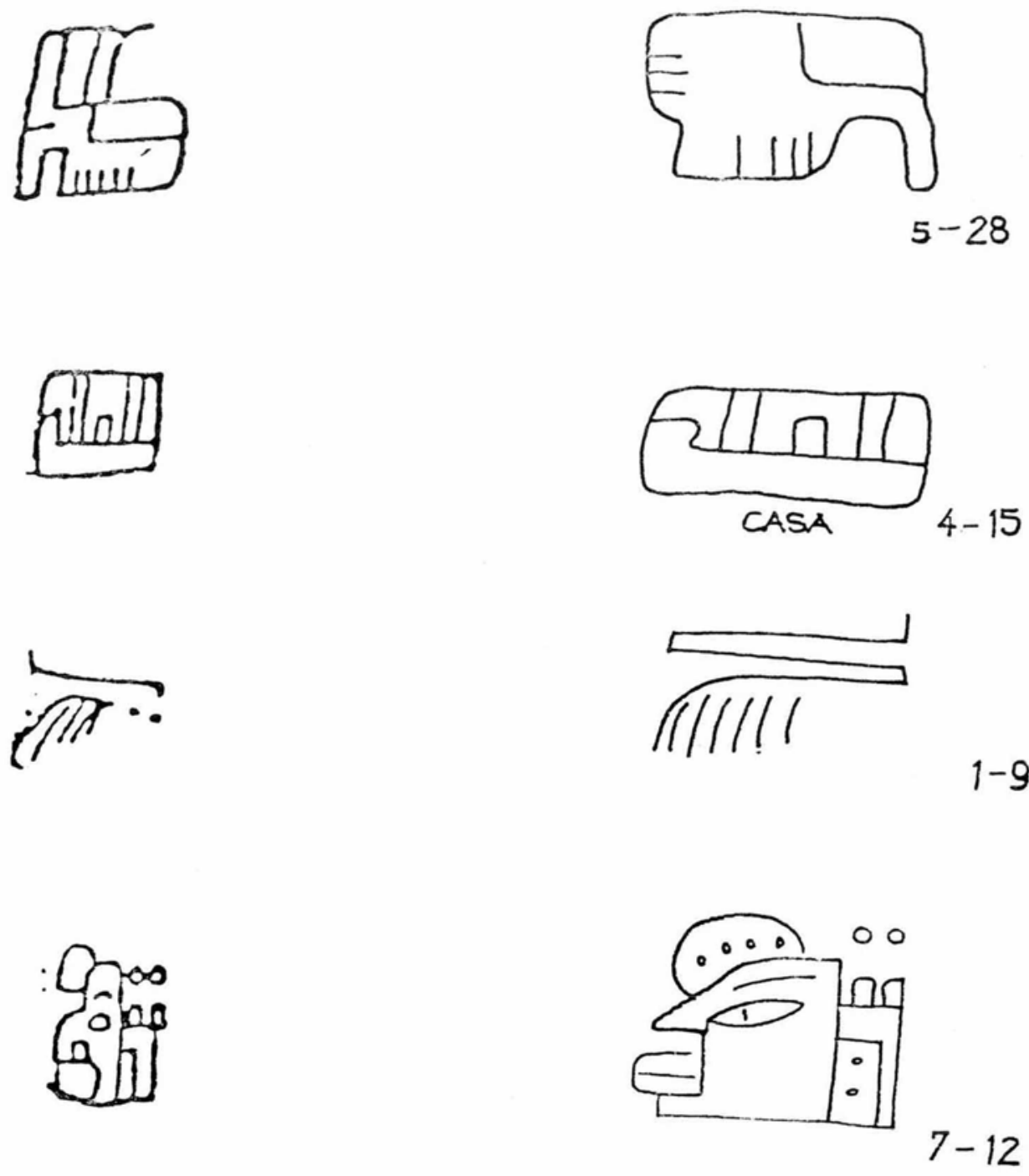

LÁMINA 1 


$$
\text { 타윽 }
$$

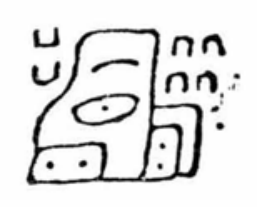
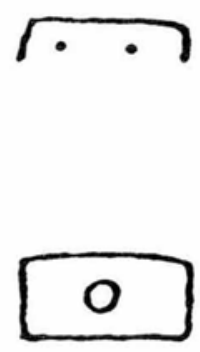
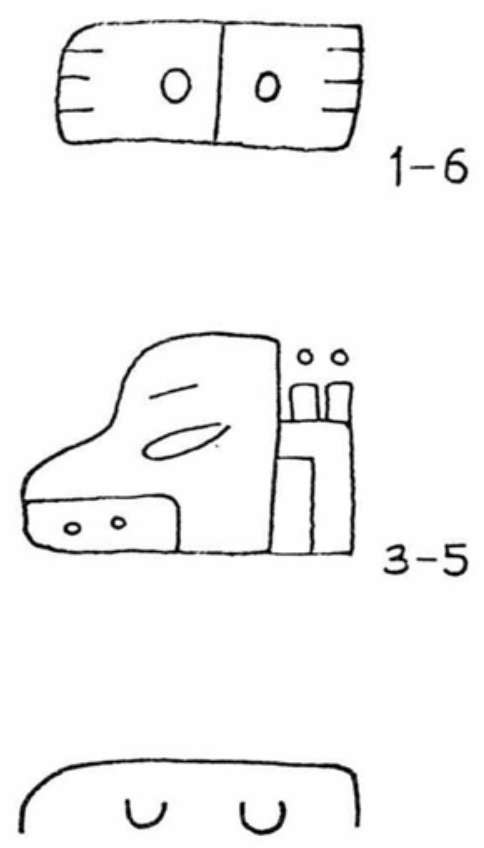

$2-14$

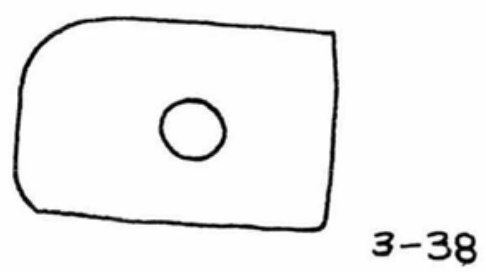

LÁMINA 2 
DOI: http://dx.doi.org/10.22201/iie.18703062e.1988.59.1406

ESTATUILLA DE TUXTLA

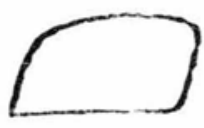

$D D$

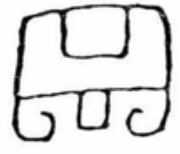

017

$\lambda$
ESTELA DE LA MOJARRA
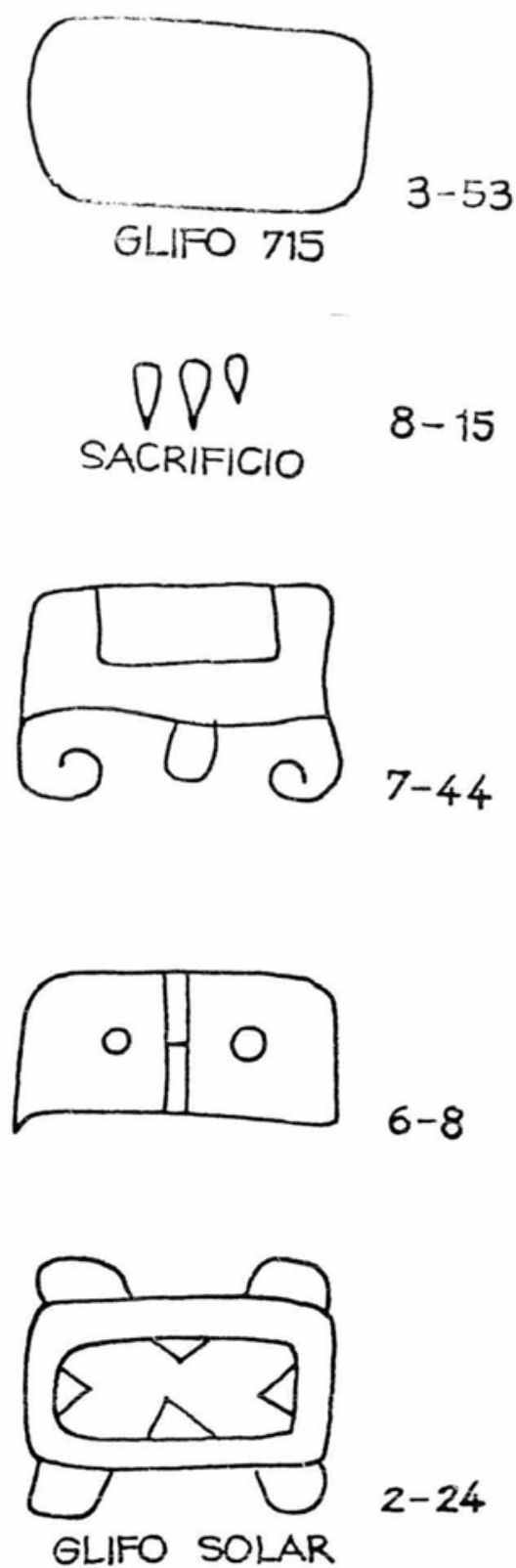

LÁMINA 3 

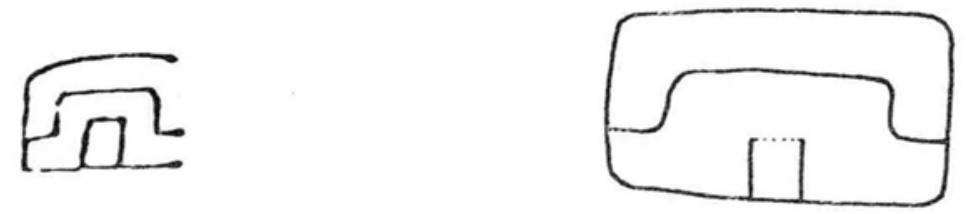

ALTAR $4-35$
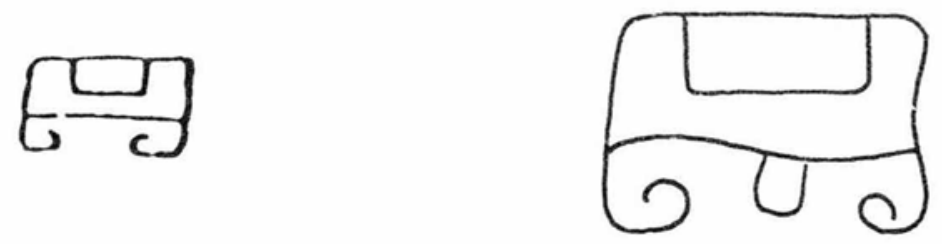

$7-44$
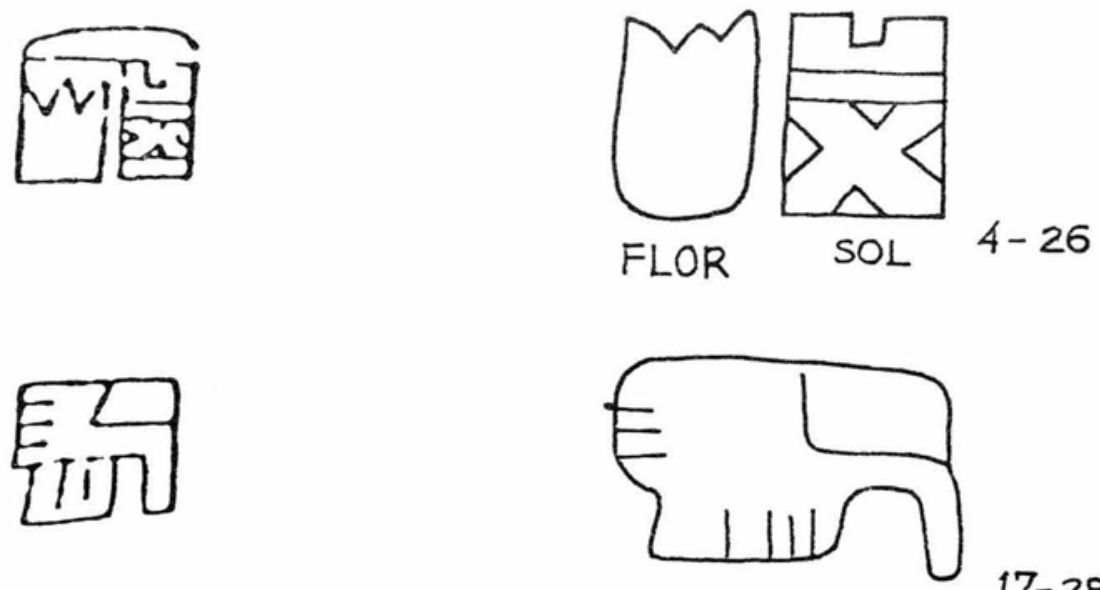

$17-28$
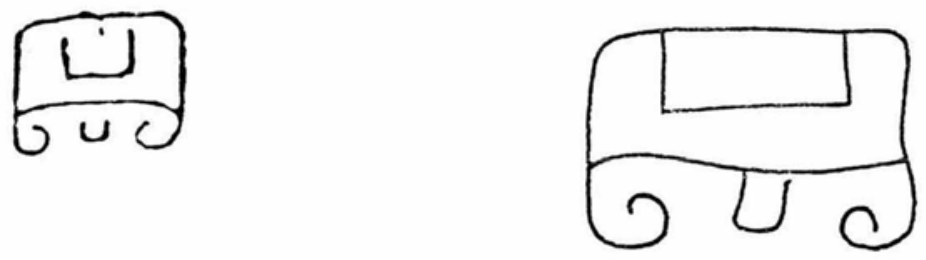

$7-44$

LÁMINA 4 

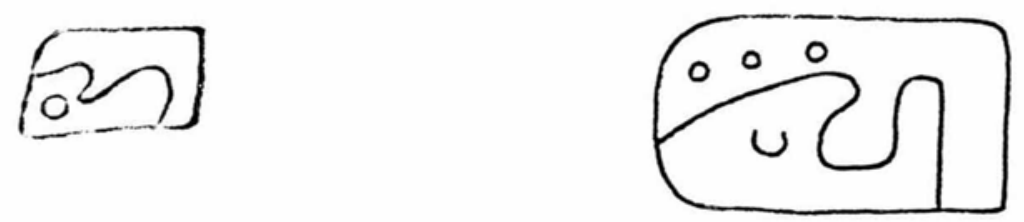

$3-28$

מ.

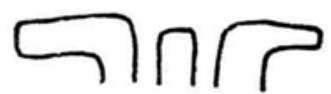

GLIFO "C" ZAPOTECA $6-50$ 
DOI: http://dx.doi.org/10.22201/iie.18703062e.1988.59.1406

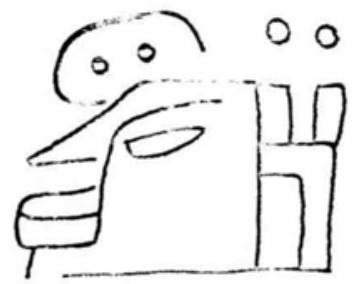

PERSONAJE
MASCUIINO
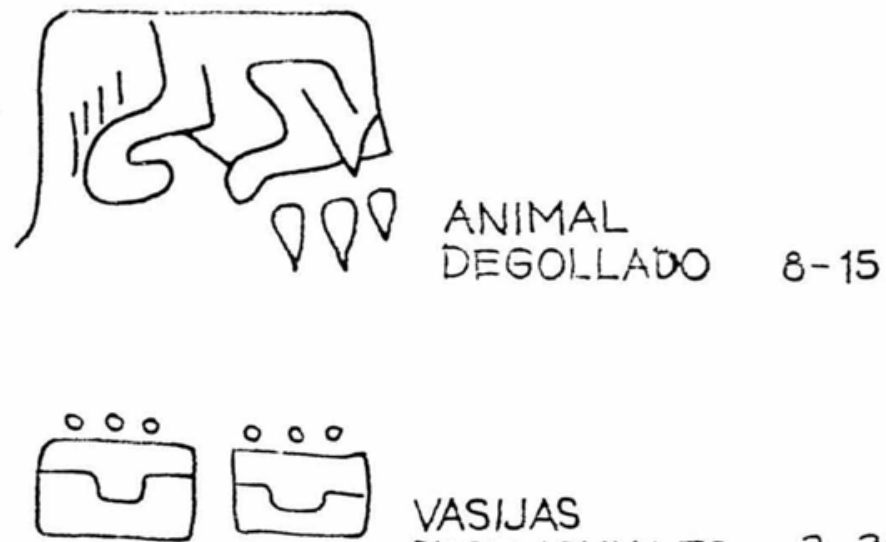

CEREMONIALES 3-39

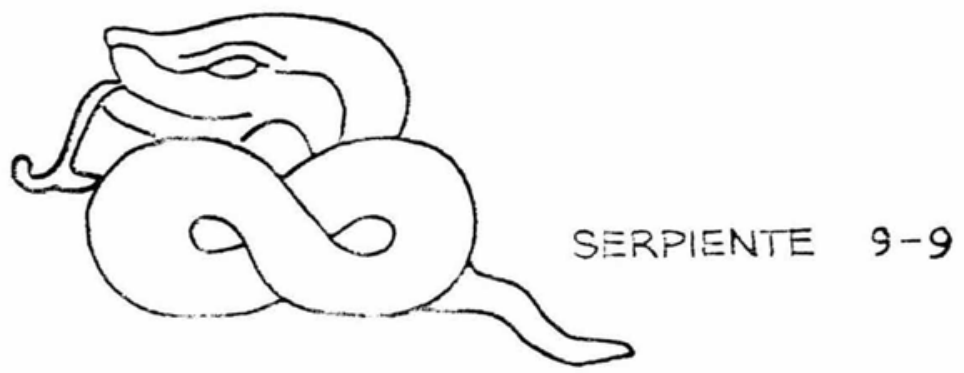

LÁMINA 6 
DOI: http://dx.doi.org/10.22201/iie.18703062e.1988.59.1406

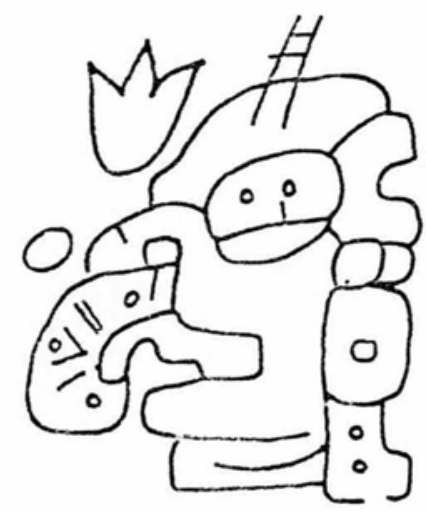

DIOS CHAAC 7-5

LAMINA 7 
DOI: http://dx.doi.org/10.22201/iie.18703062e.1988.59.1406
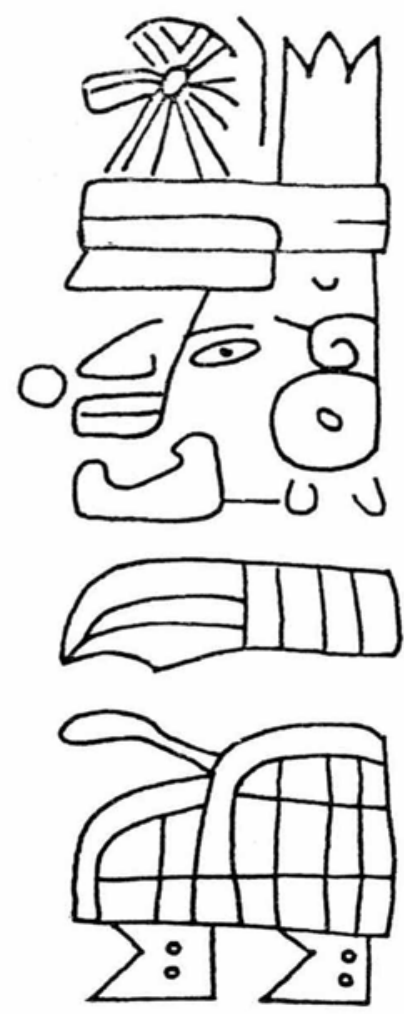

DIGNATARIO

7-9 a 11

LÁMINA 8 


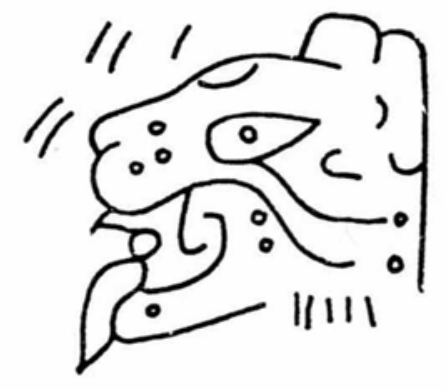

JAGUAR 4-16

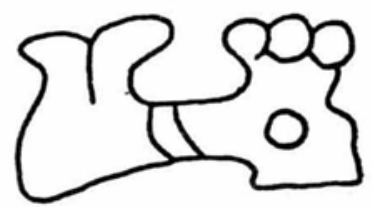

MANDÍBULA "A" $11-7$

LÁMINA 9 
DOI: http://dx.doi.org/10.22201/iie.18703062e.1988.59.1406

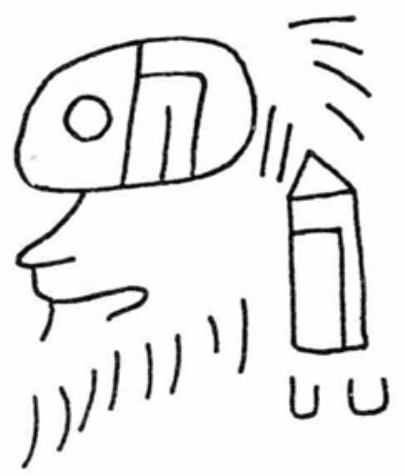

$$
2-21
$$

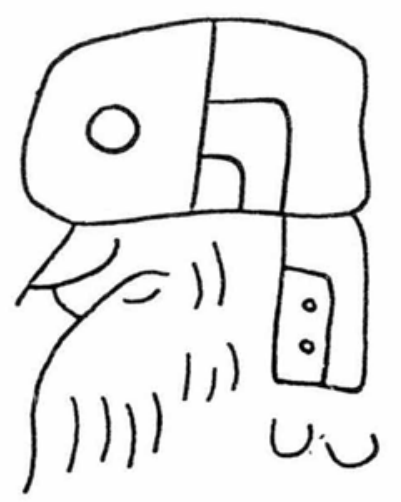

$4-12$

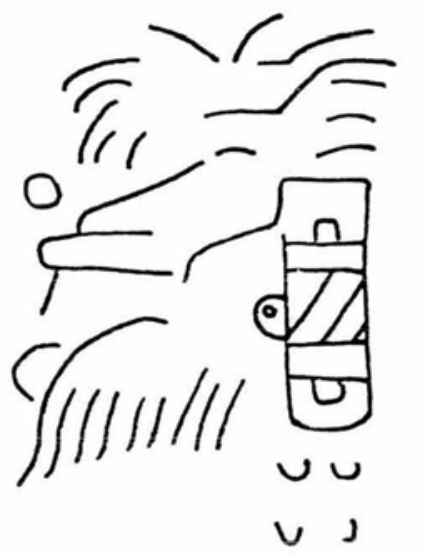

4-43

LÁMINA 10

ANCIANOS 
DOI: http://dx.doi.org/10.22201/iie.18703062e.1988.59.1406

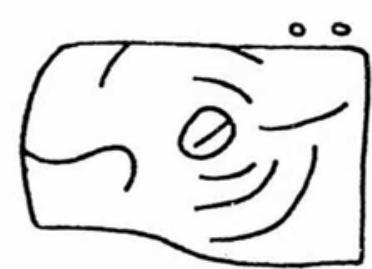

TORTUGA 3-31

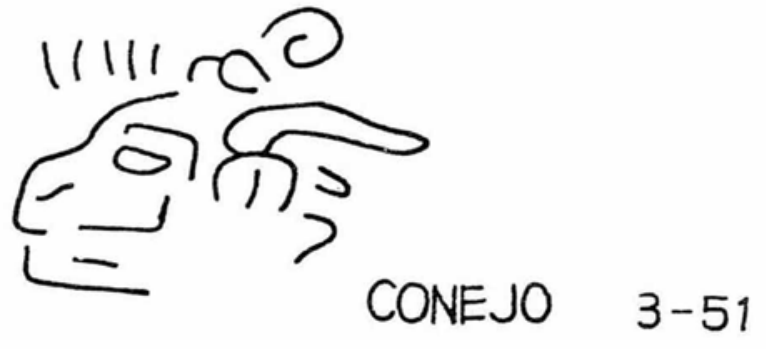

LÁMINA 11 